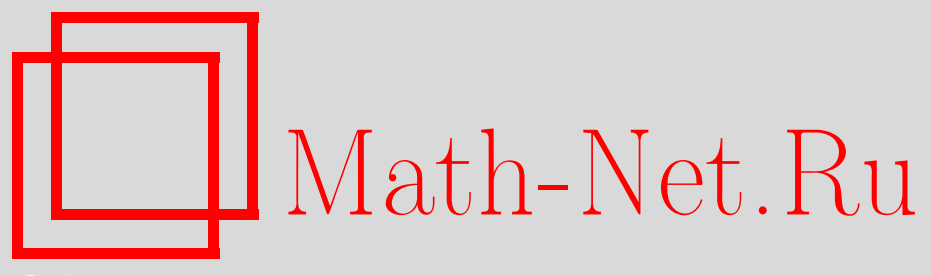

Ю. В. Егоров, О задаче Дирихле для нелинейного эллиптического уравнения, Матем. сб., 2015, том 206, номер 4, 3-12

DOI: https://doi.org/10.4213/sm8357

Использование Общероссийского математического портала Math-Net.Ru подразумевает, что вы прочитали и согласны с пользовательским соглашением http://www . mathnet.ru/rus/agreement

Параметры загрузки:

IP : 54.166 .219 .16

26 апреля 2023 г., 15:13:40

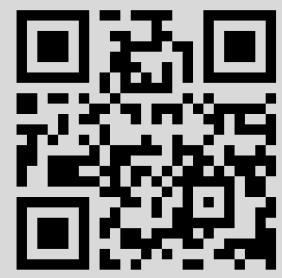


Ю. В. Егоров

\section{О задаче Дирихле для нелинейного эллиптического уравнения}

Мы доказываем существование бесконечного множества решений краевой задачи Дирихле для нелинейного эллиптического уравнения второго порядка. Такая задача для нелинейного эллиптического уравнения с оператором Лапласа изучалась ранее М. А. Красносельским, А. Бари, А. Берестицким, П. Л. Лионсом, П. Рабиновичем, М. Струве и др. Мы изучаем спектр этой задачи и доказываем слабую сходимость к 0 последовательности нормированных собственных функций. Кроме того, мы получаем оценки “коэффициентов Фурье" для функций из $W_{p, 0}^{1}(\Omega)$. Это позволяет усилить основные результаты предшествующих работ.

Библиография: 8 названий.

Ключевые слова: нелинейное эллиптическое уравнение, задача Дирихле, собственные функции.

DOI: $10.4213 / \operatorname{sm} 8357$

\section{§1. Введение}

В этой работе мы изучаем задачу Дирихле для нелинейного эллиптического уравнения второго порядка.

Пусть $\Omega$ - ограниченная область в $\mathbb{R}^{N}$ с границей $Г$ из класса $C^{1}, N>p$. Рассмотрим краевую задачу

$$
-\Delta_{p} u=|u|^{q-2} u+f(x) \quad \text { в } \Omega, \quad \Delta_{p} u \equiv \operatorname{div}\left(|\nabla u|^{p-2} \nabla u\right), \quad u=0 \quad \text { на } \Gamma,
$$

где

$$
2 \leqslant p<q<p^{*}, \quad p^{*} \equiv \frac{N p}{N-p} .
$$

Такая задача при $p=2$ изучалась ранее в работах М.А. Красносельского, А. Бари, А. Берестицкого, П.Л. Лионса, П. Рабиновича, М. Струве и др. (см. [1]-[6] и библиографии в этих работах). Основная цель этих работ состоит в доказательстве существования бесконечного числа решений для любой функции $f$ из пространства $L_{2}(\Omega)$.

В том случае, когда $f=0$, существование бесконечного числа решений при $q<p N /(N-p)$ можно вывести из теоремы Люстерника-Шнирельмана, поскольку такая задача сводится к исследованию критических точек четного функционала.

М.А. Красносельский развил теорию возмущений четного функционала (см. [1]), с помощью которой можно изучать более общую задачу (1). Его построения использованы в работе А. Бари и А. Берестицкого [2] для изучения 
задачи (1) в случае $p=2$. Наиболее сильный результат в этом направлении получен в работе А. Бари и П. Лионса [3], где доказано существование бесконечного числа решений при $p=2,1<q<(2 N-2) /(N-2)$. В работе [4] А. Бари доказал существование бесконечного числа решений при $p=2$, $1<q<2 N /(N-2)$ для множества функций $f$, которое является пересечением счетного множества всюду плотных подмножеств в пространстве $H^{-1}(\Omega)$. При этом, однако, неизвестно, содержит ли это множество хоть одну функцию из пространства $L_{2}(\Omega)$, отличную от нулевой.

Мы доказываем в этой работе слабую сходимость к нулю последовательности нормированных собственных функций данной нелинейной эллиптической краевой задачи. Это позволяет, в частности, доказать существование бесконечного множества решений у соответствующей неоднородной краевой задачи при $q \leqslant p(N-1) /(N-p)$ для функций $f$ из пространства $L_{2}(\Omega)$. Кроме того, мы доказываем существование бесконечного множества решений задачи (1) при $q<p N /(N-p)$ для всех функций $f$ из пространства $W_{p, 0}^{1}(\Omega)$.

Отметим, что в силу известного тождества С.И. Похожаева при $p=2$, $q \geqslant 2 N /(N-2)$ решение не существует, если область является звездной относительно некоторой своей точки. То же верно при $p>2, q \geqslant p N /(N-p)$, что было доказано в работе М. Отани [7].

\section{§ 2. Нелинейные собственные функции}

Положим

$$
J[u]=\int_{\Omega}\left(\frac{1}{p}|\nabla u|^{p}-\frac{1}{q}|u|^{q}\right) d x, \quad I[u]=J[u]-\int_{\Omega} f u d x .
$$

Как известно, критические точки функционала $I$ в пространстве $W_{p, 0}^{1}(\Omega)$ являются решениями задачи (1).

Теорема 1. Если $2 \leqslant p<q<p^{*}$, то существует такая последовательность критических точек $v_{n}$ функционала $J$ в пространстве $W_{p, 0}^{1}(\Omega)$, что $J\left[v_{n}\right]=b_{n} \rightarrow \infty$. Существуют такие положительные числа $C_{1}, C_{2}$, что для всех $n \in \mathbb{N}$

$$
C_{1} n^{\frac{p q}{(q-p) N}} \leqslant b_{n} \leqslant C_{2} n^{\frac{p q}{(q-p) N}} .
$$

ДокАзАтельство. Легко видеть, что для каждого $u \in W_{p, 0}^{1}(\Omega)$, не равного 0, мы имеем

$$
\sup _{t>0} J(t u)=\left(\frac{1}{p}-\frac{1}{q}\right) F[u], \quad F[u] \equiv \frac{\left(\int_{\Omega}|\nabla u|^{p} d x\right)^{\frac{q}{q-p}}}{\left(\int_{\Omega}|u|^{q} d x\right)^{\frac{p}{q-p}}} .
$$

Критические значения функционала $J$ в пространстве $W_{p, 0}^{1}(\Omega)$ взаимно однозначно соответствуют критическим значениям функционала $F$ на единичной сфере в пространстве $W_{p, 0}^{1}(\Omega)$. 
Существование бесконечной последовательности критических точек $u_{n}$ для $F$ на единичной сфере в $W_{p, 0}^{1}(\Omega)$ может быть доказано с помощью конструкции Люстерника-Шнирельмана. Значения $b_{n}=F\left[u_{n}\right]$ оцениваются ниже с помощью результатов работы А. Бари и П. Лионса [3] и метода сравнения, как это делается в книге [8] Куранта-Гильберта.

Пусть $M_{k}$ - это множество всех образов $C=g\left(S^{k}\right)$ на единичной сфере $S$ пространства $W_{p, 0}^{1}(\Omega)$, где $g$ - нечетное непрерывное отображение единичной сферы $S^{k}$ пространства $\mathbb{R}^{k+1}$ в $S$ такое, что $g(-x)=-g(x)$.

Пусть

$$
b_{k}=\inf _{C \in M_{k}} \max _{u \in C} F[u] .
$$

Покажем, что критические значения $b_{k}$ изолированы. Если $b=b_{k}$ - одно из этих значений и $u=u_{k}$ - соответствующая нормированная собственная функция, то

$$
-\Delta_{p} u=\lambda|u|^{q-2} u, \quad \int_{\Omega}|u|^{q} d x=1, \quad \int_{\Omega}|\nabla u|^{p} d x=\lambda=b^{\frac{q-p}{q}} .
$$

Если $b$ не изолировано, то существует последовательность $b_{n}$ критических значений, сходящаяся к $b$, и последовательность $u_{n}$ соответствующих собственных функций такие, что

$$
-\Delta_{p} u_{n}=\lambda_{n}\left|u_{n}\right|^{q-2} u_{n}, \quad \int_{\Omega}\left|u_{n}\right|^{q} d x=1, \quad \int_{\Omega}\left|\nabla u_{n}\right|^{p} d x=\lambda_{n}=b_{n}^{\frac{q-p}{q}} .
$$

Можно показать, что существует предел

$$
\lim _{n \rightarrow \infty} \frac{u_{n}-u}{\lambda_{n}-\lambda}=v
$$

и $v=0$ на $Г$, причем

$$
\begin{gathered}
p \int_{\Omega}|\nabla u|^{p-2} \nabla u \nabla v d x=1, \quad \int_{\Omega}|u|^{q-2} u v d x=0, \\
-(p-1) \operatorname{div}\left(|\nabla u|^{p-2} \nabla v\right)=\lambda(q-1)|u|^{q-2} v+|u|^{q-2} u .
\end{gathered}
$$

Умножая последнее уравнение на $u$ и интегрируя по $\Omega$, мы получаем, что

$$
(p-1) \int_{\Omega}|\nabla u|^{p-2} \nabla u \nabla v d x=\lambda(q-1) \int_{\Omega}|u|^{q-2} u v d x+\int_{\Omega}|u|^{q} d x .
$$

Следовательно,

$$
\int_{\Omega}|u|^{q} d x=1-\frac{1}{p}
$$

что невозможно.

Заметим, что все сказанное выше остается справедливым, если краевое условие $u=0$ заменить условием Неймана $\partial u / \partial \nu=0$ на $\Gamma$.

Рассмотрим функционал

$$
F_{\omega}[u]=\frac{\left(\int_{\omega}|\nabla u|^{p} d x\right)^{\frac{q}{q-p}}}{\left(\int_{\omega}|u|^{q} d x\right)^{\frac{p}{q-p}}}, \quad u \in W_{p, 0}^{1}(\omega),
$$


где $\omega \subset \Omega$. Положительные критические значения $b_{k}(\omega)$ этого функционала достигаются на решениях уравнения

$$
-\Delta_{p} u=\lambda_{k}|u|^{q-2} u \quad \text { в } \omega, \quad u=0 \quad \text { на } \partial \omega,
$$

для которых

$$
\int_{\omega}|\nabla u|^{p} d x=1, \quad \int_{\omega}|u|^{q} d x=\lambda_{k}^{-1}, \quad \lambda_{k}=b_{k}(\omega)^{\frac{q-p}{p}} .
$$

Из определения видно, что если $\omega_{1} \subset \omega_{2}$, то

$$
b_{k}\left(\omega_{1}\right) \geqslant b_{k}\left(\omega_{2}\right)
$$

для всех натуральных $k$.

Рассмотрим множество кубов $Q_{h}$ с длиной ребра $h$, покрывающих $\mathbb{R}^{N}$, образованных плоскостями $x_{j}=k_{j} h, j=1, \ldots, N, k_{j} \in \mathbb{Z}$. Пусть $\Omega^{\prime}$ - подмножество $\Omega$, состоящее из точек всех кубов $Q_{1}, \ldots, Q_{m}$, лежащих целиком в $\Omega$. Пусть $\Omega_{0}^{\prime}$ - открытое множество, полученное из $\Omega^{\prime}$ отбрасыванием точек, принадлежащих плоскостям $x_{j}=k_{j} h, j=1, \ldots, N, k_{j} \in \mathbb{Z}$. Мы имеем $|\Omega|=m h^{N}+o(1)$ для малых $h$.

Для каждого куба $Q_{i}$ минимальное критическое значение функционала $F$ в $W_{p, 0}^{1}\left(Q_{i}\right)$ равно

$$
c_{h}=c h^{N-\frac{p q}{q-p}},
$$

где $c$ - соответствующее минимальное критическое значение в единичном кубе $Q_{0}$. Поэтому, если $u=0$ в $r$ кубах $Q_{i}, r>0$, а в каждом из остальных такова, что на ней достигается минимальное критическое значение $b_{h}$ в пространстве $W_{p, 0}^{1}\left(Q_{i}\right)$ для этого куба, то

$$
F[u]=\frac{\left(r \int_{Q_{1}}|\nabla u|^{p} d x\right)^{\frac{q}{q-p}}}{\left(r \int_{Q_{1}}|u|^{q} d x\right)^{\frac{p}{q-p}}}=r c_{h} .
$$

Из определения легко выводятся следующие неравенства:

$$
b_{m}\left(\Omega_{0}^{\prime}\right) \geqslant b_{m}\left(\Omega^{\prime}\right) \geqslant b_{m}(\Omega) .
$$

Поэтому

$$
b_{m}(\Omega) \leqslant m c h^{N-\frac{p q}{q-p}} \leqslant C m^{\frac{p q}{N(q-p)}} .
$$

Так как $m$ может быть произвольным вместе с $h$, оценка сверху доказана. Для доказательства оценки снизу рассмотрим функционал

$$
F_{2}[u] \equiv \frac{\left(\int_{\Omega}|\nabla u|^{2} d x\right)^{\frac{q}{q-2}}}{\left(\int_{\Omega}|u|^{q} d x\right)^{\frac{2}{q-2}}} .
$$


Пусть $c_{n}$ - критические значения функционала $F_{2}$. А. Бари и П. Лионс доказали в статье [3] оценку

$$
c_{n} \geqslant C_{1} n^{\frac{2 q}{(q-2) N}} .
$$

По неравенству Гёльдера

$$
F[u] \geqslant F_{2}[u]^{\frac{q-2}{2} \frac{p}{q-p}}
$$

Поэтому для всех $n$

$$
b_{n} \geqslant c_{n}^{\frac{q-2}{2} \frac{p}{q-p}} \geqslant C_{2} n^{\frac{p q}{(q-p) N}} .
$$

Теорема доказана.

Теорема 2. Пусть $1<p<q, 2 \leqslant q<p^{*}$. Существует такая бесконечная последовательность $\left\{v_{n}\right\}$ критических точек функиионала $F$, что

$$
F\left[v_{n}\right]=b_{n} \rightarrow \infty, \quad\left\|v_{n}\right\|_{L_{2}(\Omega)}=1, \quad G\left[v_{n}\right] \leqslant C b_{n}^{\frac{1}{q}-\frac{1}{p}},
$$

где

$$
G[v] \equiv \frac{\|v\|_{W_{p, 0}^{-1}(\Omega)}}{\|v\|_{L_{q}(\Omega)}},
$$

$u$

$$
\|v\|_{W_{p, 0}^{-1}(\Omega)} \equiv \sup _{g \in W_{p, 0}^{1}(\Omega) \backslash\{0\}} \frac{\int_{\Omega} v g d x}{\|g\|_{W_{p, 0}^{1}(\Omega)}} .
$$

ДокАЗАтЕльство. Пусть $b_{n}(\omega)$ положительные критические значения функционала

$$
F_{\omega}[u]=\frac{\left(\int_{\omega}|\nabla u|^{p} d x\right)^{\frac{q}{q-p}}}{\left(\int_{\omega}|u|^{q} d x\right)^{\frac{p}{q-p}}}, \quad u \in W_{p, 0}^{1}(\omega),
$$

где $\omega \subset \Omega$.

Рассмотрим множество кубов $Q_{h}$ с длиной ребра $h$, покрывающих $\mathbb{R}^{N}$ и образованных плоскостями $x_{j}=k_{j} h, j=1, \ldots, N, k_{j} \in \mathbb{Z}$. Пусть $\Omega^{\prime}$ - подмножество $\Omega$, состоящее из точек всех кубов $Q_{1}, \ldots, Q_{m}$, лежащих целиком в $\Omega$. Пусть $\Omega_{0}^{\prime}$ - открытое множество, полученное из $\Omega^{\prime}$ отбрасыванием точек, принадлежащих плоскостям $x_{j}=k_{j} h, j=1, \ldots, N, k_{j} \in \mathbb{Z}$. Мы имеем $|\Omega|=m h^{N}+o(1)$ для малых $h$.

Для каждого куба $Q_{i}$ минимальное критическое значение функционала $F$ в $W_{p, 0}^{1}\left(Q_{i}\right)$ равно

$$
b_{h}=b h^{N-\frac{p q}{q-p}},
$$

где $b$ - соответствующее минимальное критическое значение в единичном кубе $Q_{0}$. При этом соответствующее значение функционала $F$ равно

$$
d_{h}=d h^{1+N\left(1-\frac{1}{q}-\frac{1}{p}\right)},
$$

где $d$ - соответствующее значение в единичном кубе $Q_{0}$. 
Пусть $B(\lambda, \omega), \omega \subset \Omega,-$ это число критических значений $b_{k}$ функционала $F_{\omega}$ в пространстве $W_{p, 0}^{1}(\omega)$, не превосходящих $\lambda$ и таких, что для функции $u$, на которой это значение достигается, выполнено неравенство

$$
G[u] \leqslant C F[u]^{\frac{1}{q}-\frac{1}{p}} .
$$

Если $\omega_{1}$ и $\omega_{2}-$ это подобласти $\omega_{0}$, которые не пересекаются, то

$$
B\left(\lambda, \omega_{1}\right)+B\left(\lambda, \omega_{2}\right) \leqslant B\left(\lambda, \omega_{0}\right),
$$

где $B\left(\lambda, \omega_{j}\right)$ - это число критических значений $b_{k}$ функционала $F$ в пространстве $W_{p, 0}^{1}\left(\omega_{j}\right)$, меньших $\lambda, j=0,1,2$, и таких, что для функций $u_{j}$, на которых эти значения достигаются, выполнены неравенства

$$
G\left[u_{j}\right] \leqslant C F\left[u_{j}\right]^{\frac{1}{q}-\frac{1}{p}} .
$$

В самом деле, неравенство (2) выполняется, поскольку в левой части участвуют собственные функции, равные нулю на границах множеств $\omega_{1}$ и $\omega_{2}$, а в правой части присутствуют собственные функции, равные нулю только на границе множества $\omega_{0}$.

Отсюда следует, что

$$
\sum_{i=1}^{m} B\left(\lambda, Q_{i}\right) \leqslant B(\lambda, \Omega) .
$$

Если $u=0$ в $m-r$ кубах $Q_{i}, 0<r \leqslant m$, а в каждом из остальных такова, что на ней достигается минимальное критическое значение $b_{h}$ в пространстве $W_{p, 0}^{1}\left(Q_{i}\right)$ для этого куба, то

$$
F[u]=\frac{\left(r \int_{Q_{1}}|\nabla u|^{p} d x\right)^{\frac{q}{q-p}}}{\left(r \int_{Q_{1}}|u|^{q} d x\right)^{\frac{p}{q-p}}}=b_{r}=r b_{h}, \quad G[u]=d_{r}=r^{1-\frac{1}{q}-\frac{1}{p}} d_{h} .
$$

Проверим, что для этих значений выполнены неравенства

$$
G[u] \leqslant C F[u]^{\frac{1}{q}-\frac{1}{p}} .
$$

Имеем

$$
b_{r}=r b_{0} h^{N-\frac{p q}{q-p}}, \quad d_{r}=d_{0} r^{1-\frac{1}{q}-\frac{1}{p}} h^{1+N\left(1-\frac{1}{q}-\frac{1}{p}\right)} .
$$

Надо проверить, что

$$
d_{r} \leqslant C_{1} b_{r}^{\frac{1}{q}-\frac{1}{p}} \quad \text { для } \quad 1 \leqslant r \leqslant m=C h^{-N}
$$

или

$$
r^{1-\frac{1}{q}-\frac{1}{p}} h^{1+N\left(1-\frac{1}{q}-\frac{1}{p}\right)} \leqslant C_{2} r^{\frac{1}{q}-\frac{1}{p}} h^{\left(\frac{1}{p}-\frac{1}{q}\right)\left(\frac{p q}{q-p}-N\right)} .
$$

Последнее неравенство эквивалентно неравенству

$$
r^{1-\frac{1}{q}-\frac{1}{p}+\frac{1}{p}-\frac{1}{q}} \leqslant C_{3} h^{-N\left(1-\frac{1}{q}-\frac{1}{p}+\frac{1}{p}-\frac{1}{q}\right)},
$$

которое выполнено, поскольку $r \leqslant C h^{-N}, q>2$. 
Если $\lambda \leqslant b h^{N-\frac{p q}{q-p}}$, то для каждого куба $Q_{i}$ может существовать только одно положительное критическое значение, меньшее или равное $\lambda$, так что

$$
B(\lambda, \Omega) \geqslant m \geqslant c_{1}|\Omega| h^{-N}
$$

для малых $h$, и $c_{1}>0$. Более того, $|\Omega|=m h^{N}+o(1)$ при $h \rightarrow 0$. Следовательно,

$$
b_{m} \leqslant C h^{N-\frac{p q}{q-p}} \leqslant C_{1} m^{\frac{p q}{(q-p) N}-1} .
$$

Таким образом, мы имеем $B(\lambda, \Omega) \geqslant m$, а так как $m$ выбрано произвольно, теорема доказана.

Теорема 3. Пусть $1<p<q, 2 \leqslant q<p^{*}$. Существует такая бесконечная последовательность решений $\left\{v_{n}\right\}$ краевой задачи

$$
-\Delta_{p} v_{n}=\lambda_{n}\left|v_{n}\right|^{q-2} v_{n} \quad \text { в } \Omega, \quad v_{n}=0 \quad \text { на } \Gamma,
$$

чmo $\lambda_{n+1}>\lambda_{n} u$

$$
\left\|v_{n}\right\|_{L_{2}(\Omega)} \leqslant C,
$$

причем $\lambda_{n} \rightarrow \infty u\left\{v_{n}\right\}$ слабо сходится $к 0$ в $L_{2}(\Omega)$, когда $n \rightarrow \infty$.

Доказательство. Результат сразу следует из теоремы 2 и следующего критерия слабой сходимости в гильбертовом пространстве.

Лемма 1. Пусть $H$ - сепарабельное гильбертово пространство $u\left\{u_{n}\right\}$ некоторая бесконечная последовательность элементов пространства mаких, что $\left\|u_{n}\right\| \leqslant C$. Последовательность $\left\{u_{n}\right\}$ слабо сходится $\kappa$ 0, т.е. $\left(f, u_{n}\right) \rightarrow 0$ при $n \rightarrow \infty$ для любого $f \in H$, тогда и толъко тогда, когда $\left(u_{n}, u_{k}\right) \rightarrow 0$ при $n \rightarrow \infty$ для каждого натурального $k$.

ДокАзАТЕльство. Необходимость условия следует из определения слабой сходимости.

Для доказательства достаточности рассмотрим подпространство $H^{\prime}$ пространства $H$, порожденное элементами $\left\{u_{n}\right\}$. В этом пространстве линейные комбинации элементов $\left\{u_{n}\right\}$ образуют всюду плотное подмножество. Для каждого элемента $v$ этого подмножества имеем $\left(v, u_{n}\right) \rightarrow 0$ при $n \rightarrow \infty$. Следовательно, $\left\{u_{n}\right\}$ слабо сходится к 0 в $H^{\prime}$, а потому и в $H$.

\section{§ 3. Неоднородная краевая задача}

Теорема 4. Пусть $2 \leqslant p<N, p<q \leqslant \frac{p(N-1)}{N-p} u f \in L_{2}(\Omega)$. Тогда краевая задача (1) имеет бесконечное множество решений.

Доказательство. Решения краевой задачи (1) - это критические точки функционала $I$. Функционал $J$ имеет бесконечное множество таких критических точек $u_{n}$, что $b_{n} \equiv J\left[u_{n}\right] \rightarrow \infty$. По теореме 1 имеем

$$
b_{n} \geqslant C_{1} n^{\frac{p q}{(q-p) N}} .
$$


Поэтому существует такая постоянная $C>0$, что для бесконечного множества значений $n$ справедливо неравенство

$$
\left|b_{n+1}-b_{n}\right|>C b_{n}^{1-\frac{N(q-p)}{p q}}
$$

(см., например, [2; лемма 5.3]). Поскольку

$$
\left|\int_{\Omega} f u_{n} d x\right|<c_{1} b_{n}^{\frac{1}{q}}
$$

где $c_{1}$ не зависит от $n$, тогда если выполнено неравенство

$$
\frac{1}{q}<1-\frac{N(q-p)}{p q}
$$

или, эквивалентно, $q(N-p)<p(N-1)$, то существует критическое значение функционала $I$ в $\left(b_{n}, b_{n+1}\right)$.

Если

$$
q=\frac{p(N-1)}{N-p}
$$

то мы используем слабую сходимость. Именно, положим

$$
v_{n}=\left[\frac{q-p}{p q b_{n}}\right]^{1 / q} u_{n}
$$

так что $\left\|v_{n}\right\|_{L_{q}(\Omega)}=1$ и

$$
-\Delta_{p} v_{n}=\lambda_{n}\left|v_{n}\right|^{q-2} v_{n} \quad \text { в } \Omega, \quad v_{n}=0 \quad \text { на } \quad \Gamma, \quad \lambda_{n}=\left[\frac{p q b_{n}}{q-p}\right]^{\frac{q-p}{q}} \text {. }
$$

По теореме 3 последовательность $v_{n}$ слабо сходится к 0 в $L_{2}(\Omega)$ при $n \rightarrow \infty$. Следовательно, для любого $\varepsilon>0$ имеем

$$
\left|\int_{\Omega} f(x) v_{n}(x) d x\right|<\varepsilon
$$

если $n>n_{0}$ и $n_{0}$ достаточно велико. Поэтому

$$
\left|\int_{\Omega} f(x) u_{n}(x) d x\right|<c \varepsilon b_{n}^{1 / q},
$$

если $n>n_{0}$, с постоянной $c$, не зависящей от $n$. Как показано в работе [2], из теоремы 1 следует, что для бесконечного множества значений $n$ мы имеем

$$
\left|J\left[u_{n}\right]-J\left[u_{n+1}\right]\right|>C b_{n}^{1 / q}
$$

с постоянной $C>0$, не зависящей от $n$, и поэтому существует критическое значение функционала $I$ между $b_{n}$ и $b_{n+1}$. Теорема доказана. 
ЗАмечание 1. А. Бари и П. Л. Лионс доказали в [3], что теорема 4 верна при

$$
p=2, \quad 1<q<\frac{2 N-2}{N-2} .
$$

В этом случае мы добавляем только значение

$$
q=\frac{2 N-2}{N-2} .
$$

Из теоремы 1 видно, что этим методом нельзя продвинуться дальше (хотя возможно, что теорема 4 верна при $\left.q<p^{*}\right)$.

Теорема 5. Пусть $2 \leqslant p<N, p<q<\frac{N p}{N-p} u f \in W_{p, 0}^{1}(\Omega)$. Тогда краевая задача (1) имеет бесконечное множество решений.

ДокАзАтельство. Решения краевой задачи (1) - это критические точки функционала $I$. Функционал $J$ имеет бесконечное множество таких критических точек $u_{n}$, что $b_{n} \equiv J\left[u_{n}\right] \rightarrow \infty$. Поскольку по теореме 2

$$
\left|\int_{\Omega} f(x) u_{n}(x) d x\right| \leqslant\|f\|_{W_{p, 0}^{1}(\Omega)}\left\|u_{n}\right\|_{W_{p, 0}^{-1}(\Omega)} \leqslant C b_{n}^{\frac{1}{q}-\frac{1}{p}}
$$

для бесконечного множества значений $n$, и для бесконечного множества значений $n$ справедливо неравенство

$$
\left|b_{n+1}-b_{n}\right|>C b_{n}^{1-\frac{N(q-p)}{p q}}
$$

a

$$
\frac{1}{q}-\frac{1}{p}<0<1-\frac{N(q-p)}{p q},
$$

тогда для бесконечного множества значений $n$ существует критическое значение функционала $I$ в $\left(b_{n}, b_{n+1}\right)$. Теорема доказана.

\section{Список литературы}

[1] М. А. Красносельский, Топологические методь в теории нелинейных интегральных уравнений, Гостехиздат, М., 1956, 392 с.; англ. пер.: M. A. Krasnosel'skii, Topological methods in the theory of nonlinear integral equations, A Pergamon Press Book The Macmillan Co., New York, 1964, xi+395 pp.

[2] A. Bahri, H. Berestycki, "A perturbation method in critical point theory and applications", Trans. Amer. Math. Soc., 267:1 (1981), 1-32.

[3] A. Bahri, P. L. Lions, "Morse index of some min-max critical points. I. Application to multiplicity results", Comm. Pure Appl. Math., 41:8 (1988), 1027-1037.

[4] A. Bahri, "Topological results on a certain class of functionals and applications", J. Funct. Anal., 41:3 (1981), 397-427.

[5] M. Struwe, "Infinitely many critical points for functionals which are not even and applications to superlinear boundary value problems", Manuscripta Math., 32:3-4 (1980), 335-364. 
[6] P.H. Rabinowitz, "Multiple critical points of perturbated symmetric functionals", Trans. Amer. Math. Soc., 272:2 (1982), 753-769.

[7] M. Ôtani, "A remark on certain nonlinear elliptic equations", Proc. Fac. Sci. Tokai Univ., 19 (1984), 23-28.

[8] Р. Курант, Д. Гильберт, Методы математической физики, т. 1, ГТТИ, М.-Л., 1933, 528 с.; пер. с нем.: R. Courant, D. Hilbert, Methoden der mathematischen Physik, v. I, Grundlehren Math. Wiss., 12, J. Springer, Berlin, 1931, xiv+469 pp.

\section{Юрий Владимирович Егоров} (Yuriy V. Egorov)

Поступила в редакцию Institute de Mathématique de Toulouse, France 10.03.2014 и 12.09.2014

E-mail: egorov@cegetel.net 